\title{
An extremely rare case of radiculous premolar coexisting with bilateral four- rooted mandibular second molars.
}

\author{
Dimitar Yovchev $^{1 *}$, Hristina Mihaylova ${ }^{1}$, Angela Gusiyska ${ }^{2}$, Nikoleta Traykova ${ }^{3}$, Vladimir Nikolov ${ }^{4}$, \\ Nadezhda Miteva Yovcheva ${ }^{5}$ \\ ${ }^{1}$ Department of Imaging and Oral Diagnostics, Faculty of Dental Medicine, Medical University, Sofia, Bulgaria \\ ${ }^{2}$ Department of Conservative Dentistry, Faculty of Dental Medicine, Medical University, Sofia, Bulgaria \\ ${ }^{3}$ Department of Diagnostic Imaging, University Hospital "St George" JSC, Plovdiv, Bulgaria \\ ${ }^{4}$ Department of Diagnostic imaging, Military Medical Academy, Sofia, Bulgaria \\ ${ }^{5}$ Serdika Hospital, Sofia, Bulgaria
}

\begin{abstract}
We present a rare case of three-rooted left permanent maxillary first premolar (MFP) coexisting with bilateral four-rooted permanent mandibular second molars (MSM). These variants were found incidentally on cone beam computed tomography (CBCT) images. At best of our knowledge, this is the first report presenting these rare tooth root variants in one patient. Although this condition is uncommon the radiologists and clinicians should be aware because of its importance for the successful root canal treatment, future tooth extraction, apicectomy and other procedures. This report adds a new knowledge about tooth roots variants and illustrates the possibility of their coexistence in one patient.
\end{abstract}

Keywords: Cone-beam computed tomographic imaging, Mandibular molar, Maxillary premolar, Root variants.

\section{Introduction}

One of the main reasons for endodontic treatment failures is the lack of understanding or underestimation of the root canal morphology [1].

The intraoral radiographs are usually useful to see the number, length, curvature and possible variations of the roots and their canals. But the visualization of some variants like three root canals of the MFPs with preoperative radiography is not always possible [2-4].

Recently CBCT with an optimal field of view is an effective method of choice to diagnose the tooth roots and the canals morphology due to higher resolution than traditional computed tomography and the lower radiation $[4,5]$.

Typically, the maxillary first premolar (MFP) has two roots and two canals, but the number of roots and the canals can vary from one to three. The incidence of three canals ranges from $1.2 \%$ to $6 \%$ [6-8]. In case of three canals in a premolar they can be distributed in one, two or three roots [9]. The term "radiculous" is used to describe the three-rooted premolars.

Usually, the mandibular second molar (MSM) is described as having three canals, two in the mesial and one in the distal root, but the number of the root canals can vary up to five, mostly located in two roots [10-13]. In the MSM, each root consists of two radicals which usually are not separated along the root. In some cases the two radicals can be separated apically, but complete separation is rare [14]. Respectively up to four roots in permanent MSM are described [1,15-20].

Morphological dental anomalies can be related to a single tooth, group of teeth or may affect the entire dentition [21-23].

Separate cases of three-rooted MFPs and four-rooted MSMs have been reported [7-9,11,16,17,20,24]. To best of our knowledge a coexistence of these variants in one patient has not been reported yet.

\section{Case Description}

We present a case of 63-y-old Caucasian female underwent $\mathrm{CBCT}$ in order to evaluate both jaws before implant treatment planning.

The CBCT scan was taken by CBCT (ILUMA ${ }^{\mathrm{TM}}$, Imtec Imaging, Ardmore, OK). It was performed according to the manufacturer's recommended exposure settings $(120 \mathrm{KV}, 40 \mathrm{~s}$, $3.8 \mathrm{~mA}$ ) and patient positioning protocol.

The patient had given informed consent for this examination and the case description was carried out in accordance with the principles described in the Declaration of Helsinki and all 
amendments and revisions. Only the investigators had access to the patient data.

On the CBCT images a three-rooted left MFP with two incompletely divided buccal radicals and one palatal root was found incidentally (Figure 1).

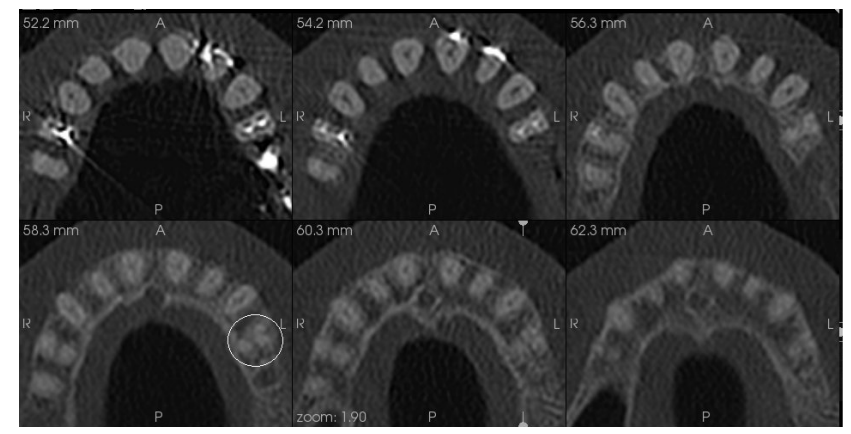

Figure 1. CBCT axial slices presenting three-rooted left MFP.

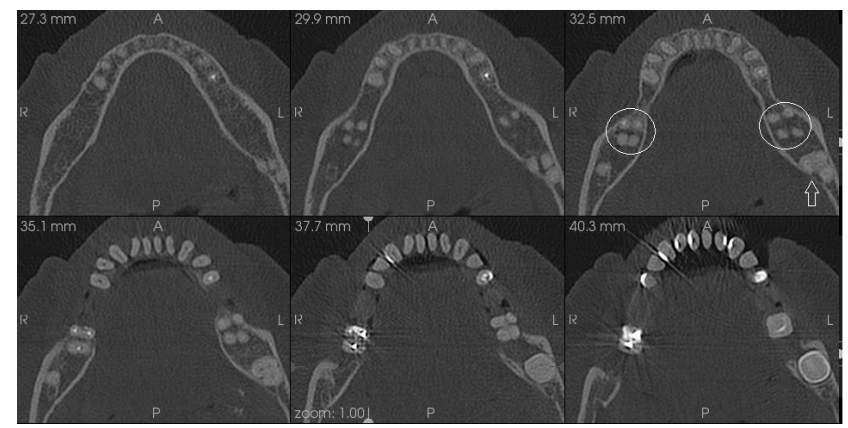

Figure 2. CBCT axial slices of the same patient presenting bilateral four-rooted MSMs and impacted 38 (empty arrow).

Additionally, MSMs with well separated radicals $(n=4)$ forming four roots (two mesial and two distal) were found bilaterally. An impacted left lower third molar was also seen during the radiographic examination (Figure 2).

\section{Discussion}

The knowledge about the teeth root and canal variations is very important for the successful endodontic treatment and endodontic surgery. Sometimes these variants can be incidental findings on the imaging studies used in dentistry as in the presented case.

In order to diagnose a three-rooted maxillary premolar on plain $\mathrm{x}$ ray image a rule proposed by Sieraski et al. can be used. They found that when the mesio-distal width of the mid-root image is equal or greater than the width of the tooth's crown, the tooth is most likely to be with three roots [25]. Vertical or horizontal angulation of the x-ray tube can help to avoid the root overlapping and in case of doubts CBCT can be used as a method of choice to confirm the number of roots and their canals $[3,4]$.

The symmetry of roots and root's canal morphology is common for MSM as in our patient, although Plotino et al. found asymmetrical occurrence in $20 \%$ of cases [13].
The left three-rooted MFP in the presented case was unilateral and respectively asymmetric regarding the root number in comparison with the opposite two-rooted MFP.

The incidence of three-rooted MFP is not very rare findingVertucci et al. found 5\% of 400 MFP to have three canals: $0.5 \%$ are teeth with three canals in a single root, $0.5 \%$ have three canals in two roots and $4 \%$ have one canal in each of the three separate roots [9]. But Carns et al. found 6\% of MFP $(n=100)$ to have three roots with a one canal in each of them [6].

In contrast, the presence of four rooted MSM is a very rare finding- $0.55 \%$ according to the results of Shemesh et al. [20]. There are a few case reports and studies reporting data about four-rooted MSM, among them Purra et al. described an interesting case of MSM with three mesial and one distal root $[11,16,20,24]$.

In our case three-rooted MFP coexists with bilateral fourrooted MSM and we didn't find a report or study describing such a combination.

This case emphasize on the possibility of coexistence in one patient of two rare dental root anatomy variations (except commonly seen impaction of 38). Therefore clinicians should pay attention if one anatomic variant is present and to be aware for coexistence with another.

Further investigations should be performed regarding the incidence of such combinations between these rare but important anatomic variants.

\section{Conclusions}

CBCT can be very useful tool to present the morphology of the roots and their canals especially in case of doubt.

The whole field of view must be carefully observed and all found variations have to be notified.

Although coexistence of three-rooted MFP with four-rooted MSM is uncommon the radiologists and clinicians should be aware because of its importance for the dental practice.

This report adds a new knowledge about tooth roots variants and illustrates the possibility of their coexistence in one patient.

\section{Conflict of Interests}

The authors declare that they have no conflict of interests.

\section{References}

1. Thakar SS, Motghare V, Prabhakar I, Shivlingesh KK, Gupta B, Gupta N. Bilateral presence of a single root in mandibular second molars having a single non-conical canal configuration: a rare case report. Int J Adv Health Sci 2014; 1: 31-34. 
2. Kirilova J, Topalova-Pirinska S, Kirov D. Variation of maxillary first premolar with three root canals. J IMAB 2014; 20: 584-588.

3. Cooke HG III, Cox FL. C-shaped canal configurations in mandibular molars. J Am Dent Assoc 1979; 99: 836-839.

4. Zhang R, Wang H, Tian YY, Yu X, Hu T, Dummer PM. Use of cone-beam computed tomography to evaluate root and canal morphology of mandibular molars in Chinese individuals. Int Endod J 2011; 44: 990-999.

5. Tu MG, Huang HL, Hsue SS, Hsu JT, Chen SY, Jou MJ. Detection of permanent three-rooted mandibular first molars by cone-beam computed tomography imaging in Taiwanese individuals. J Endod 2009; 35: 503-507.

6. Carns EJ, Skidmore AE. Configurations and deviations of root canals of maxillary first premolars. Oral Surg 1973; 36: $880-886$.

7. Karumaran CS, Gunaseelan R, Krithikadatta J. Microscopeaided endodontic treatment of maxillary first premolars with three roots: A case series. Ind J Dent Res 2011; 22: 706-708.

8. Kumar A, Iftekhar H, Andrabi SM. Endodontic management of a three rooted maxillary first premolar. Guindent 2012; 5: 32-33.

9. Vertucci FJ, Gegauff A. Root canal morphology of the maxillary first premolar. J Am Dent Assoc 1979; 99:194-198.

10. Beatty RG, Krell K. Mandibular molars with five canals: report of two cases. J Am Dent Assoc 1987; 114: 802.

11. Peiris R, Pitakotuwage N, Takahashi M, Ohzeki S, Nakayama M, Sakurai S, Igarashi Y, Matsuno M, Sasaki K, Satake T, Kanazawa E. Mandibular permanent second molar with four roots and root canals: a case report. Odontology 2009; 97: 51-53.

12. Pineda F, Kuttler Y. Mesiodistal and buccolingual roentgenographic investigation of 7275 root canals. Oral Surg Oral Med Oral Pathol Oral Radiol Endod 1972; 33: 101-110.

13. Plotino G, Tocci L, Grande NM, Testarelli L, Messineo D, Ciotti M, Glassman G, D'ambrosio F, Gambarini G. Symmetry of root and root canal morphology of maxillary and mandibular molars in a white population: a cone-beam computed tomography study in vivo. J Endod 2013; 39: 1545-1548.

14. Carlsen O. Dental morphology (1st ed). Munksgaard, Copenhagen, Denmark 1987.

15. Manning SA. Root canal anatomy of mandibular second molars (Part I). Int Endod J 1990; 23: 34-39.

16. Martins JNR, Ascenso J, Caramês G. Endodontic treatment of a mandibular second molar with four roots-A case report and literature review. Giornale Italiano di Endodonzia 2014; 28: 23-28.

17. Park JB, Kim N, Park S, Kim Y, Ko Y. Evaluation of root anatomy of permanent mandibular premolars and molars in Korean population with cone-beam computed tomography. Eur J Dent 2013; 7: 94-101.

18. Rahimi S, Shahi S, Lofti M, Zand , Abdolrahimi M, Eshaghi R. Root canal configuration and prevalence of Cshaped canal in mandibular second molars in an Iranian population. J Oral Sci 2008; 50: 9-13.

19. Sharma D. Single rooted mandibular second molars with single canal: rare occurrence. J Dentofac Sci 2013; 2: 27-30.

20. Shemesh A, Levin A, Katzenell V, Itzhak JB, Levinson O, Zini A, Solomonov M. Prevalence of 3 and 4-rooted first and second mandibular molars in the Israeli population. $\mathbf{J}$ Endodontics 2015; 41: 338-342.

21. Metgud S, Metgud R, Rani K. Management of a patient with a taurodont, single-rooted molars associated with multiple dental anomalies: a spiral computerized tomography evaluation. Oral Surg Oral Med Oral Pathol Oral Radiol Endod 2009; 108: 81-86.

22. Nguyen AH, Tiffee JC, Arnold RM. Pyramidal molar roots and canine-like morphologic features in multiple family members - a case report. Oral Surg Oral Med Oral Pathol Oral Radiol Endod 1996; 82: 411-416.

23. Robbins IM, Keene HJ. Multiple morphologic dental anomalies-report of a case. Oral Surg Oral Med Oral Pathol Oral Radiol Endod 1964; 17: 683-690.

24. Purra AR, Mushtaq M, Robbani I, Farooq R. Spiral computed tomographic evaluation and endodontic management of a mandibular second molar with four roots. A case report and literature review. Iran Endod J 2013; 8: 69-71.

25. Sieraski SM, Taylor GT, Kohn RA. Identification and endodontic management of three-canalled maxillary premolars. J Endod 1985; 15: 29-32.

\section{*Correspondence to}

Dimitar Yovchev

Department of Imaging and Oral Diagnostics

Faculty of Dental Medicine

Medical University

Sofia

Bulgaria 